Supporting Information for

\title{
Force Field Benchmark of Amino Acids. 3. Hydration with Scaled Lennard-Jones Interactions
}

\author{
Yejie Qiu, Wenjie Shan, and Haiyang Zhang*
}

Department of Biological Science and Engineering, School of Chemistry and Biological Engineering, University of Science and Technology Beijing, 100083 Beijing, China

\section{Corresponding Author}

* zhanghy@ustb.edu.cn 
Table S1. Scaling Parameters Tested for Computing Hydration Free Energies of Amino Acid Side Chains

\begin{tabular}{|c|c|c|c|c|}
\hline force field & strategy $^{a}$ & water $^{b}$ & cutoff $^{c}$ & scaling parameters $^{d}$ \\
\hline Amber ff14SB & I & TIP3P & 10 & $\gamma=1.00,1.05,1.09,1.10,1.15$ \\
\hline CHARMM36m & I & TIP3P & 10 & $\gamma=1.00,1.05,1.09,1.10,1.15$ \\
\hline CHARMM36m & I & TIP3P & 12 & $\gamma=1.00,1.05,1.10,1.15,1.20$ \\
\hline CHARMM36m & I & TIPS3P & 10 & $\gamma=1.00,1.05,1.09,1.10,1.15$ \\
\hline CHARMM36m & I & TIPS3P & 12 & $\gamma=1.00,1.05,1.10,1.15,1.20$ \\
\hline OPLS-AA/M & I & TIP4P & 10 & $\gamma=1.00,1.05,1.09,1.10,1.15$ \\
\hline Amber ff14SB & II & TIP3P & 10 & $\sigma_{\mathrm{H}}=0.400014, \varepsilon_{\mathrm{H}}=0.005,0.01,0.025,0.046,0.069$ \\
\hline CHARMM36m & II & TIP3P & 10 & $\sigma_{\mathrm{H}}=0.400014, \varepsilon_{\mathrm{H}}=0.025,0.046,0.069,0.1,0.125,0.15$ \\
\hline CHARMM36m & II & TIP3P & 12 & $\sigma_{\mathrm{H}}=0.400014, \varepsilon_{\mathrm{H}}=0.046,0.1,0.15,0.2,0.25$ \\
\hline CHARMM36m & II & TIPS3P & 10 & $\sigma_{\mathrm{H}}=0.400014, \varepsilon_{\mathrm{H}}=0.025,0.046,0.069,0.1,0.125,0.15$ \\
\hline CHARMM36m & II & TIPS3P & 12 & $\sigma_{\mathrm{H}}=0.400014, \varepsilon_{\mathrm{H}}=0.046,0.1,0.15,0.25,0.3$ \\
\hline OPLS-AA/M & II & TIP4P & 10 & $\sigma_{\mathrm{H}}=0.400014, \varepsilon_{\mathrm{H}}=0.046,0.1,0.25,1.0,2.5,5.0$ \\
\hline OPLS-AA/M & II & TIP4P & 10 & $\sigma_{\mathrm{H}}=1.239203, \varepsilon_{\mathrm{H}}=0.005,0.0155,0.025,0.046,0.069,0.1,0.155$ \\
\hline Amber ff14SB & III & TIP3P & 10 & $\Delta \mathrm{C}_{6}=0 \%,-4 \%,-10 \%$ \\
\hline CHARMM36m & III & TIP3P & 10 & $\Delta \mathrm{C}_{6}=0 \%,-4 \%,-10 \%$ \\
\hline CHARMM36m & III & TIP3P & 12 & $\Delta \mathrm{C}_{6}=0 \%,-4 \%,-10 \%$ \\
\hline CHARMM36m & III & TIPS3P & 10 & $\Delta \mathrm{C}_{6}=0 \%,-4 \%,-10 \%$ \\
\hline CHARMM36m & III & TIPS3P & 12 & $\Delta \mathrm{C}_{6}=0 \%,-4 \%,-10 \%$ \\
\hline OPLS-AA/M & III & TIP4P & 10 & $\Delta \mathrm{C}_{6}=0 \%,-4 \%,-10 \%$ \\
\hline CHARMM36m & IV & TIPS3P & 10 & $\sigma_{\mathrm{H}}=0, \varepsilon_{\mathrm{H}}=0, \gamma=1.00,1.10,1.15,1.20,1.25$ \\
\hline CHARMM36m & IV & TIPS3P & 12 & $\sigma_{\mathrm{H}}=0, \varepsilon_{\mathrm{H}}=0, \gamma=1.00,1.05,1.10,1.15,1.20,1.25,1.30$ \\
\hline
\end{tabular}

Table S2. System Setup for the MD Simulations of Folded and Intrinsically Disordered Proteins

\begin{tabular}{llrlrll}
\hline protein & PDB code & box $\left(\AA^{3}\right)$ & $\begin{array}{c}\text { ionic concentration (mol/L) } \\
\text { folded proteins }\end{array}$ & $N_{\text {water }}$ & $N_{\mathrm{Na}+}$ & $N_{\mathrm{Cl}-}$ \\
GB3 & 1P7E & $50 \times 50 \times 50$ & 0 & 3869 & 2 & 0 \\
BPTI & 5PTI & $50 \times 50 \times 50$ & 0 & 3849 & 0 & 7 \\
ubiquitin & 1D3Z & $50 \times 50 \times 50$ & 0.15 & 3724 & 11 & 11 \\
\multicolumn{2}{c}{ intrinsically disordered proteins (IDPs) } \\
A 340 & 1BA4 & $60 \times 60 \times 60$ & 0.05 & 6946 & 10 & 7 \\
CspTm & 1G6P & $60 \times 60 \times 60$ & 0.1 & 6784 & 13 & 13 \\
IN & 1WJB & $60 \times 60 \times 60$ & 0.1 & 6854 & 15 & 13 \\
Protein L & 2PTL & $60 \times 60 \times 60$ & 0.1 & 6735 & 22 & 13 \\
\hline
\end{tabular}

Table S3. Lennard-Jones Parameters Used for the Plotting in Figure 3b.

\begin{tabular}{llll}
\hline atom & $\sigma_{\mathrm{i}}(\AA)$ & $\varepsilon_{\mathrm{i}}(\mathrm{kcal} / \mathrm{mol})$ & note \\
oplsm_272 & 0.0296 & 0.21 & from OPLS-AA/M \\
OW & 3.15365 & 0.155 & oxygen in TIP4P \\
HW & 0.400014 & 0.046 & hydrogen in TIPS3P \\
HWc & 1.239203 & 0.0155 & $\sigma_{\mathrm{i}}$ is computed by eq $6 ; \varepsilon_{\mathrm{i}}$ is $10 \%$ of TIP4P oxygen \\
\hline
\end{tabular}


Table S4. Lennard-Jones Parameters of the Oxygen Atom in the Water Models Tested in This Work and the Intermolecular Energies $\left(E_{\mathrm{i}}, \mathrm{kcal} / \mathrm{mol}\right)$ of Water Molecules

\begin{tabular}{|c|c|c|c|c|c|c|c|}
\hline model & & $\begin{array}{c}C_{6} \\
\left(10^{-3} \mathrm{~kJ} \mathrm{~mol}^{-1} \mathrm{~nm}^{6}\right)\end{array}$ & $\begin{array}{c}C_{12} \\
\left(10^{-6} \mathrm{~kJ} \mathrm{~mol}^{-1} \mathrm{~nm}^{12}\right)\end{array}$ & $\begin{array}{c}\sigma \\
(\mathrm{nm})\end{array}$ & $\begin{array}{c}\varepsilon \\
(\mathrm{kJ} / \mathrm{mol})\end{array}$ & $\begin{array}{c}\text { LJ } \\
\text { cutoff }(\mathrm{nm})\end{array}$ & $E_{\mathrm{i}}$ \\
\hline \multirow[t]{8}{*}{3 -site } & OPC3 & 2.79758 & 2.86185 & 0.31743 & 0.68369 & 1.0 & -11.76 \\
\hline & $\mathrm{SPC} / \mathrm{E}$ & 2.61882 & 2.63522 & 0.31656 & 0.65063 & 1.0 & -11.19 \\
\hline & $\mathrm{SPC} / \mathrm{E}_{\mathrm{b}}$ & 2.61882 & 2.63522 & 0.31656 & 0.65063 & 1.0 & -11.76 \\
\hline & TIP3P & 2.48971 & 2.43510 & 0.31506 & 0.63639 & 1.0 & -9.59 \\
\hline & & & & & & 1.2 & -9.51 \\
\hline & TIPS3P & 2.48971 & 2.43510 & 0.31506 & 0.63639 & 1.0 & -9.87 \\
\hline & & & & & & 1.2 & -9.79 \\
\hline & TIP3P-FB & 2.68716 & 2.76811 & 0.31780 & 0.65214 & 1.0 & -11.78 \\
\hline \multirow[t]{7}{*}{ 4-site } & a99SB-disp & 4.01664 & 4.03743 & 0.31650 & 0.99899 & 1.0 & -12.69 \\
\hline & OPC & 3.59040 & 3.61961 & 0.31666 & 0.89036 & 1.0 & -12.31 \\
\hline & TIP4P & 2.55190 & 2.51041 & 0.31537 & 0.64852 & 1.0 & -9.91 \\
\hline & TIP4P/2005 & 3.07965 & 3.05997 & 0.31589 & 0.77487 & 1.0 & -11.43 \\
\hline & TIP4P-D & 3.76727 & 3.78677 & 0.31650 & 0.93697 & 1.0 & -12.22 \\
\hline & TIP4P-Ew & 2.73451 & 2.74528 & 0.31644 & 0.68095 & 1.0 & -11.11 \\
\hline & TIP4P-FB & 3.01577 & 3.03455 & 0.31656 & 0.74928 & 1.0 & -11.94 \\
\hline exp. & & $2.61918^{a}$ & & & & & $-9.92^{b}$ \\
\hline
\end{tabular}

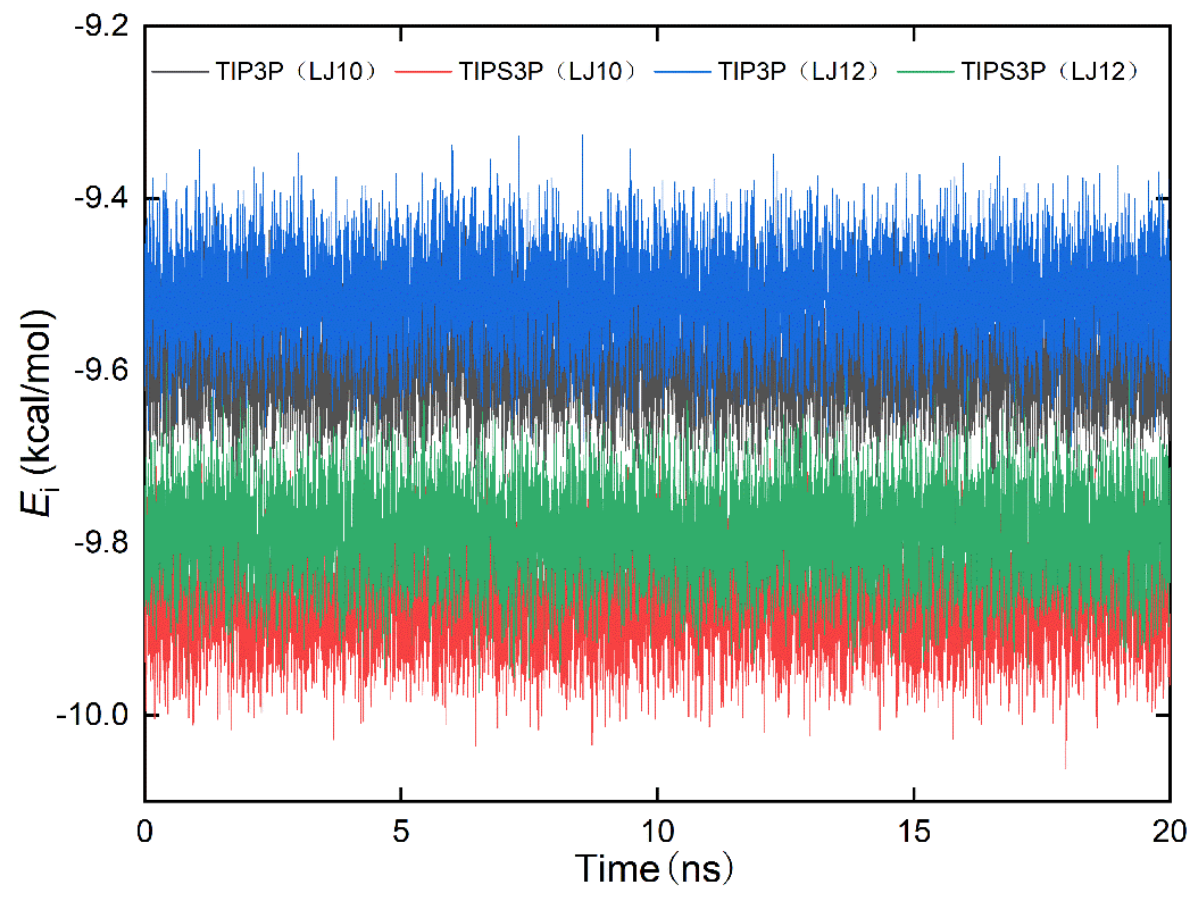

Figure S1. Interaction energies of water molecules $\left(E_{\mathrm{i}}\right)$ as a function of simulation time with different water models (TIP3P and TIPS3P) and Lennard-Jones (LJ) cutoff (10 and $12 \AA$ ). 

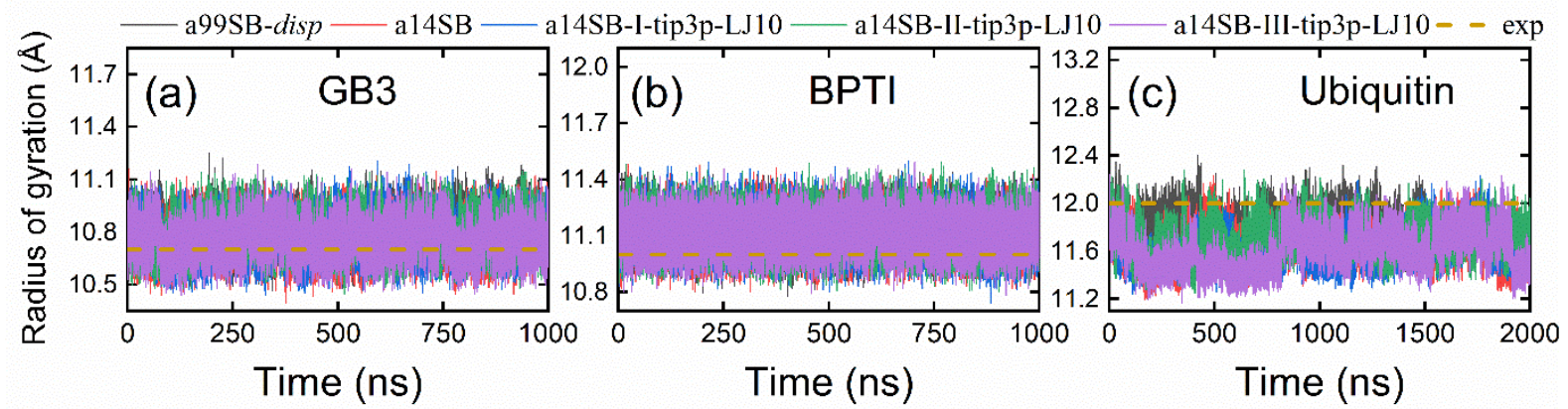

Figure S2. Radius of gyration as a function of simulation time for the folded proteins of the third IgG-binding domain of Protein G (GB3, a), bovine pancreatic trypsin inhibitor (BPTI, b), and ubiquitin (c) with original and scaled Lennard-Jones (LJ) parameters. The legends of a99SB-disp and a14SB mean the use of original a99SB-disp and Amber ff14SB force fields, respectively. Dashed orange lines indicate experimental observations of crystal structures. The other legends and LJ scaling parameters used in the strategies I-III are given in Table 2 in the main text.
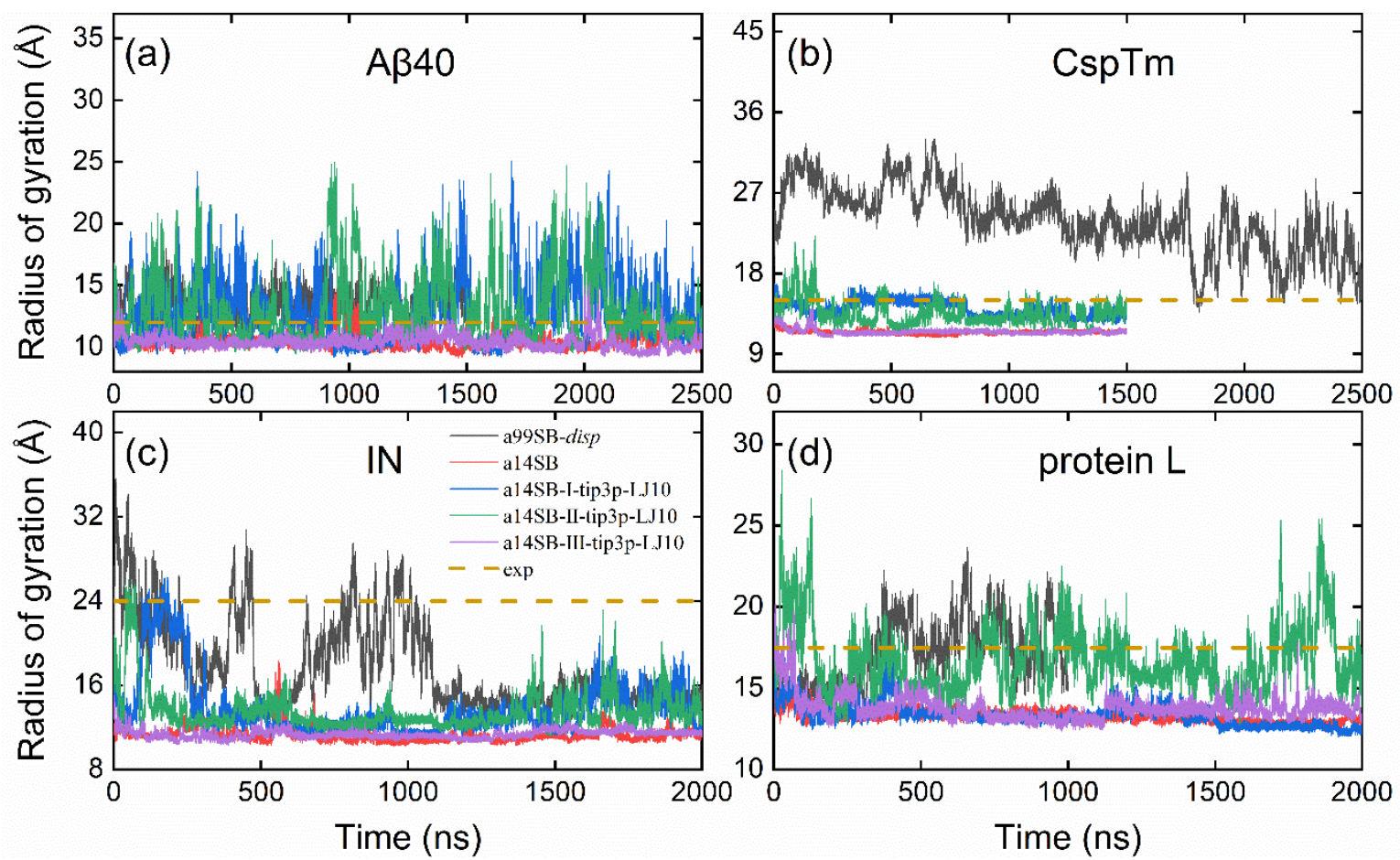

Figure S3. Radius of gyration as a function of simulation time for intrinsically disordered proteins of A $\beta 40$ (a), the cold-shock protein from Thermotoga maritima (CspTm, b), HIV-1 integrase (IN, c), and immunoglobulin-binding domain of protein L (d) with original and scaled Lennard-Jones (LJ) parameters. The legends of a99SB-disp and a14SB mean the use of original a99SB-disp and Amber ff14SB force fields, respectively. Dashed orange lines indicate experimental observations. The other legends and LJ scaling parameters used in the strategies I-III are given in Table 2 in the main text. 


\section{References}

(1) Zeiss, G. D.; Meath, W. J. Dispersion Energy Constants C 6(a, B), Dipole Oscillator Strength Sums and Refractivities for Li, N, O, $\mathrm{H}_{2}, \mathrm{~N}_{2}, \mathrm{O}_{2}, \mathrm{NH}_{3}, \mathrm{H}_{2} \mathrm{O}, \mathrm{NO}$ and $\mathrm{N}_{2} \mathrm{O}$. Mol. Phys. 1977, 33, 1155-1176.

(2) Jorgensen, W. L.; Chandrasekhar, J.; Madura, J. D.; Impey, R. W.; Klein, M. L. Comparison of Simple Potential Functions for Simulating Liquid Water. J. Chem. Phys. 1983, 79, 926-935. 